\title{
Identification problems for systems of nonlinear evolution equations and functional equations
}

\author{
Yurii E Anikonov ${ }^{1}$, İsmet Gölgeleyen² and Mustafa Yildiz²*
}

"Correspondence:

mustafa.yildiz@beun.edu.tr

${ }^{2}$ Department of Mathematics,

Faculty of Arts and Sciences, Bülent

Ecevit University, Incivez,

Zonguldak, 67100, Turkey

Full list of author information is

available at the end of the article

\begin{abstract}
In this work, we consider two inverse problems for systems of nonlinear differential and differential-difference evolution equations. We propose a new technique for the solution of such inverse problems. We reduce the problem to a system of functional equations by using the given data and then we obtain the solution by the method of Kuczma (Functional Equations in a Single Variable, 1968).
\end{abstract}

Keywords: inverse problem; system of nonlinear evolution equations; functional equation

\section{Introduction}

Systems of nonlinear evolution equations are the mathematical models that are used to describe many complex phenomena arising in science and engineering. In the last few decades, considerable progress has been made in this theory. We refer to some recent books [2-4] for a complete survey of both the theory and its applications. However, most of these works have been focused on direct problems and there have been few results on inverse problems for systems of nonlinear evolution equations. Some inverse problems for kinetic and other evolution equations are discussed in [5].

In this paper, we first consider the system of nonlinear evolution equations

$$
\frac{\partial w_{j}}{\partial t}=A_{j} w_{j}(x, t)+f_{j}(x) B_{j} w_{j}(x, t)+g_{j}\left(w_{1}, w_{2}, \ldots, w_{n}\right), \quad j=1,2, \ldots, n,
$$

in the domain $\Omega=\{(x, t):(x, t) \in D \times[a, b]\}$, where $D=\{x:|x|<\tau\}$ is an open domain in the Euclidean space $\mathbb{R}^{n}$ of variable $x=\left(x_{1}, x_{2}, \ldots, x_{n}\right), n \geq 1 ; a, b$ are fixed constants; $A_{j}, B_{j}$ are some known finite differential operators with sufficiently differentiable coefficients and act only on the variable $x$.

The principal result of this work is to reduce inverse problems for systems (1) and (20) to a system of functional equations by using only four data of previous history. It is worth remembering here the classical work in mathematical modeling where the data of previous history were used. For example, using the laws of Kepler, Isaac Newton formulated the potential of gravitation and using the laws of Faraday and Ampère, James Clerk Maxwell obtained the differential equations for the electromagnetic field. More recently, Jay Wright

(c) 2016 Anikonov et al. This article is distributed under the terms of the Creative Commons Attribution 4.0 International License (http://creativecommons.org/licenses/by/4.0/), which permits unrestricted use, distribution, and reproduction in any medium, provided you give appropriate credit to the original author(s) and the source, provide a link to the Creative Commons license, and indicate if changes were made. 
Forrester who is known as the founder of system dynamics for the world economy used the experimental data of the years 1900-1970.

\section{Main results}

We consider the following inverse problem:

Problem 1 Find the continuous functions $f_{j}(x), g_{j}(z)$ that satisfy system (1) and the data

$$
\begin{array}{ll}
\left.w_{j}(x, t)\right|_{t=a}=\varphi_{j a}(x), & \left.\frac{\partial w_{j}}{\partial t}(x, t)\right|_{t=a}=\psi_{j a}(x), \\
\left.w_{j}(x, t)\right|_{t=b}=\varphi_{j b}(x), & \left.\frac{\partial w_{j}}{\partial t}(x, t)\right|_{t=b}=\psi_{j b}(x),
\end{array}
$$

where $j=1,2, \ldots, n ; x \in D \subset \mathbb{R}^{n} ; z \in \mathbb{R}^{n}$.

Let us define the vector-functions $\varphi_{a}(x), \varphi_{b}(x), \psi_{a}(x), \psi_{b}(x)$ by the equalities

$$
\begin{aligned}
& \varphi_{a}(x)=\left(\varphi_{1 a}(x), \varphi_{2 a}(x), \ldots, \varphi_{n a}(x)\right), \quad \varphi_{b}(x)=\left(\varphi_{1 b}(x), \varphi_{2 b}(x), \ldots, \varphi_{n b}(x)\right), \\
& \psi_{a}(x)=\left(\psi_{1 a}(x), \psi_{2 a}(x), \ldots, \psi_{n a}(x)\right), \quad \psi_{b}(x)=\left(\psi_{1 b}(x), \psi_{2 b}(x), \ldots, \psi_{n b}(x)\right) .
\end{aligned}
$$

We assume that $\varphi_{a}(x), \varphi_{b}(x) \in D$ for $x \in D$ and the mapping $y=\varphi_{a}(x), x \in D, \varphi_{a}(x) \in D$ has an inverse mapping $x=\varphi_{a}^{-1}(y)$. We introduce the vector-function $\lambda(x)$ and the functionals $R_{j}(x), S_{j}(x)$ as follows:

$$
\begin{aligned}
& \lambda(x)=\varphi_{a}^{-1}\left(\varphi_{b}(x)\right), \quad R_{j}(x)=\frac{\left.B_{j} \varphi_{j a}(y)\right|_{y=\lambda(x)}}{B_{j} \varphi_{j b}(x)}, \\
& S_{j}(x)=\frac{\left.A_{j} \varphi_{j a}(y)\right|_{y=\lambda(x)}-A_{j} \varphi_{j b}(x)+\psi_{j b}(x)-\psi_{j a}(\lambda(x))}{B_{j} \varphi_{j b}(x)},
\end{aligned}
$$

where $B_{j} \varphi_{j b}(x) \neq 0, j=1,2, \ldots, n$.

In this paper, we always assume that the solutions $w_{j}(x, t), j=1,2, \ldots, n$, of equations in (1) exist and the data in (2) are given such that the vector-function $\lambda(x)$ and the functions $R_{j}(x)$, $S_{j}(x)$ are at least continuous on $D$. We also note that the dimension $n$ of space $\mathbb{R}^{n}$ must be equal to the number of equations in system (1) and differentials for $x$ in the operators $A_{j}$, $B_{j}, j=1,2, \ldots, n$.

Theorem 1 If there exist constants $q_{j} \in(0,1)$ and $\alpha_{j}>0$ such that

$$
\left|R_{j}(x)\right| \leq q_{j}, \quad\left|S_{j}(x)\right| \leq \alpha_{j}, \quad j=1,2, \ldots n, x \in D,
$$

then the solution of Problem 1 is given by the following formulas:

$$
\begin{aligned}
& f_{j}(x)=\sum_{k=0}^{\infty} \prod_{s=0}^{k-1} R_{j}\left(\lambda^{[s]}(x)\right) S_{j}\left(\lambda^{[k]}(x)\right), \\
& g_{j}(z)=\psi_{j a}\left(\varphi_{a}^{-1}(z)\right)-\left.A_{j} \varphi_{j a}(y)\right|_{y=\varphi_{a}^{-1}(z)}-\left.f_{j}\left(\varphi_{a}^{-1}(z)\right) B_{j} \varphi_{j a}(y)\right|_{y=\varphi_{a}^{-1}(z)},
\end{aligned}
$$

where $\lambda^{[k]}(x)=\underbrace{\lambda(\lambda(\cdots(\lambda(x)) \cdots))}_{k}, \lambda^{[k+1]}(x)=\lambda\left(\lambda^{[k]}(x)\right), k=0,1,2, \ldots$, and $\lambda^{[0]}(x)=x$. 
Proof We set $t=a$ and $t=b$ in (1), respectively, then by (2), (3) we obtain

$$
\begin{aligned}
& \psi_{j a}(x)=A_{j} \varphi_{j a}(x)+f_{j}(x) B_{j} \varphi_{j a}(x)+g_{j}\left(\varphi_{a}(x)\right), \\
& \psi_{j b}(x)=A_{j} \varphi_{j b}(x)+f_{j}(x) B_{j} \varphi_{j b}(x)+g_{j}\left(\varphi_{b}(x)\right), \quad j=1,2, \ldots, n .
\end{aligned}
$$

In (6), let us replace $x$ by $\lambda(x)=\varphi_{a}^{-1}\left(\varphi_{b}(x)\right)$ to have

$$
\psi_{j a}(\lambda(x))=\left.A_{j} \varphi_{j a}(y)\right|_{y=\lambda(x)}+\left.f_{j}(\lambda(x)) B_{j} \varphi_{j a}(y)\right|_{y=\lambda(x)}+g_{j}\left(\varphi_{b}(x)\right)
$$

By (7), (8) we have

$$
\begin{aligned}
\psi_{j a}(\lambda(x))-\psi_{j b}(x)= & \left.A_{j} \varphi_{j a}(y)\right|_{y=\lambda(x)}+\left.f_{j}(\lambda(x)) B_{j} \varphi_{j a}(y)\right|_{y=\lambda(x)} \\
& -A_{j} \varphi_{j b}(x)-f_{j}(x) B_{j} \varphi_{j b}(x)
\end{aligned}
$$

in which the functions $g_{j}\left(\varphi_{b}(x)\right)$ are not included. The last equality is a functional equation of the form

$$
f_{j}(x)=R_{j}(x) f_{j}(\lambda(x))+S_{j}(x)
$$

for $f_{j}(x)$.

Numerous examples and results of the theory and applications of functional equations can be found in $[1,6]$. In order to find $f_{j}(x)$ in the functional equation (9), the following formula is presented in [6]:

$$
f_{j}(x)=\sum_{k=0}^{\infty} \prod_{s=0}^{k-1} R_{j}\left(\lambda^{[s]}(x)\right) S_{j}\left(\lambda^{[k]}(x)\right) .
$$

It is worth noting that if $R_{j}(x), S_{j}(x)$ and $\lambda(x)$ are continuous functions in the last equality, then so is $f_{j}(x)$, [6]. Moreover, if $y=\varphi_{a}^{-1}(z)$, then the function $g_{j}(z)$ can be calculated from (6) as follows:

$$
\begin{aligned}
g_{j}(z)= & \psi_{j a}\left(\varphi_{a}^{-1}(z)\right)-\left.A_{j} \varphi_{j a}(y)\right|_{y=\varphi_{a}^{-1}(z)} \\
& -\left.f_{j}\left(\varphi_{a}^{-1}(z)\right) B_{j} \varphi_{j a}(y)\right|_{y=\varphi_{a}^{-1}(z)}
\end{aligned}
$$

which completes the proof of the theorem.

Remark 1 We note that formula (4) can be written as

$$
f_{j}(x)=S_{j}(x)+\sum_{k=1}^{\infty} \prod_{s=0}^{k-1} R_{j}\left(\lambda^{[s]}(x)\right) S_{j}\left(\lambda^{[k]}(x)\right) .
$$

In fact, from the functional equation (9) we have the following relations:

$$
R_{j}(x) f_{j}(\lambda(x))=R_{j}(x) R_{j}(\lambda(x)) f_{j}\left(\lambda^{[2]}(x)\right)+R_{j}(x) S_{j}(\lambda(x))
$$




$$
\begin{aligned}
\prod_{s=0}^{k-1} R_{j}\left(\lambda^{[s]}(x)\right) f_{j}\left(\lambda^{[k]}(x)\right)= & \prod_{s=0}^{k-1} R_{j}\left(\lambda^{[s]}(x)\right) R_{j}\left(\lambda^{[k]}(x)\right) f_{j}\left(\lambda^{[k+1]}(x)\right) \\
& +\prod_{s=0}^{k-1} R_{j}\left(\lambda^{[s]}(x)\right) S_{j}\left(\lambda^{[k]}(x)\right), \quad k \geq 1,
\end{aligned}
$$

If we add (9) and (11) we obtain formula (10). Moreover, the series in (10) is convergent because from (10) by the hypothesis of Theorem 1 we have the majorizing series

$$
\alpha_{j}+q_{j} \alpha_{j}+q_{j}^{2} \alpha_{j}+\cdots=\alpha_{j}\left(1+q_{j}+q_{j}^{2}+\cdots\right)=\alpha_{j} \frac{1}{1-q_{j}} .
$$

Example 1 If we take $n=1, a=0, b=T, j=1, A_{1}(x)=\frac{\partial^{2}}{\partial x^{2}}, B_{1}(x)=\left(\frac{\partial}{\partial x}\right)^{2}, w_{j}(x, t)=w(x, t)$, $f_{j}(x)=f(x), g_{j}(z)=g(z)$ in $(1)$, then we obtain the equation

$$
\frac{\partial w}{\partial t}=\frac{\partial^{2} w}{\partial x^{2}}+f(x)\left(\frac{\partial w}{\partial x}\right)^{2}+g(w(x, t)), \quad 0 \leq x \leq \tau, 0 \leq t \leq T,
$$

with the data

$$
\begin{array}{ll}
\left.w(x, t)\right|_{t=0}=\varphi_{0}(x), & \left.\frac{\partial w}{\partial t}(x, t)\right|_{t=0}=\psi_{0}(x), \\
\left.w(x, t)\right|_{t=T}=\varphi_{T}(x), & \left.\frac{\partial w}{\partial t}(x, t)\right|_{t=T}=\psi_{T}(x),
\end{array}
$$

where $0 \leq\left|\varphi_{0}(x)\right| \leq \tau, 0 \leq\left|\varphi_{T}(x)\right| \leq \tau$.

In that case the inverse problem is to determine the functions $f(x)$ and $g(z)$ which satisfy relations (12)-(13).

Then by (12)-(13) we can have a system of equations

$$
\begin{aligned}
& \psi_{0}(x)=\varphi_{0}^{\prime \prime}(x)+f(x)\left(\varphi_{0}^{\prime}(x)\right)^{2}+g\left(\varphi_{0}(x)\right), \\
& \psi_{T}(x)=\varphi_{T}^{\prime \prime}(x)+f(x)\left(\varphi_{T}^{\prime}(x)\right)^{2}+g\left(\varphi_{T}(x)\right)
\end{aligned}
$$

for the functions $f(x)$ and $g(z)$. By replacing the variable $x$ by $\lambda(x)=\varphi_{0}^{-1}\left(\varphi_{T}(x)\right)$ in (14) and taking into account (15) we have

$$
\psi_{T}(x)-\psi_{0}(\lambda(x))=\varphi_{T}^{\prime \prime}(x)+f(x)\left(\varphi_{T}^{\prime}(x)\right)^{2}-\left.f(\lambda(x))\left(\varphi_{0}^{\prime}(y)\right)^{2}\right|_{y=\lambda(x)}-\left.\varphi_{0}^{\prime \prime}(y)\right|_{y=\lambda(x)} .
$$

The last equality is a functional equation for $f(x)$ of the form

$$
f(x)=R(x) f(\lambda(x))+S(x)
$$

where

$$
R(x)=\frac{\left.\left(\varphi_{0}^{\prime}(y)\right)^{2}\right|_{y=\lambda(x)}}{\left(\varphi_{T}^{\prime}(x)\right)^{2}}, \quad S(x)=\frac{\left.\varphi_{0}^{\prime \prime}(y)\right|_{y=\lambda(x)}-\varphi_{T}^{\prime \prime}(x)+\psi_{T}(x)-\psi_{0}(\lambda(x))}{\left(\varphi_{T}^{\prime}(x)\right)^{2}}
$$

Here we note that the functions $R(x)$ and $S(x)$ are determined only by the given data. 
Thus, if $|R(x)| \leq q<1,|S(x)| \leq \alpha, 0 \leq x \leq \tau$, then we obtain the following formulas:

$$
\begin{aligned}
& f(x)=\sum_{k=0}^{\infty} \prod_{s=0}^{k-1} R\left(\lambda^{[s]}(x)\right) S\left(\lambda^{[k]}(x)\right) \\
& g(z)=\psi_{0}\left(\varphi_{0}^{-1}(z)\right)-\left.\varphi_{0}^{\prime \prime}(y)\right|_{y=\varphi_{0}^{-1}(z)}-\left.f\left(\varphi_{0}^{-1}(z)\right)\left(\varphi_{0}^{\prime}(y)\right)^{2}\right|_{y=\varphi_{0}^{-1}(z)}
\end{aligned}
$$

Remark 2 If $S(x)=0$ in (17), then it is clear that we have $f(x)=0$ and equation (12) is called the Kolmogorov-Petrovskii-Piskunov equation, which plays a fundamental role in a great number of models of reaction-diffusion processes in biology, chemistry, genetics, and so on.

Remark 3 Let us assume that $S(x)=0$ and $R(x)=c$ in (16), where $c>1$ is a constant. Then we obtain one of the most important iterative functional equations

$$
s f(x)=f(\lambda(x)), \quad s=\frac{1}{c}
$$

which is referred to as the Schröder equation [6].

The following example is related with the case $R(x)=1$.

Example 2 We consider the inverse problem of finding the functions $f(x)$ and $g(w)$ from the relations

$$
\begin{aligned}
& \frac{\partial w(x, t)}{\partial t}=k \frac{\partial^{2} w}{\partial x^{2}}+f(x)\left(\frac{\partial w}{\partial x}\right)^{s}+g(w), \quad 0 \leq x \leq \tau, 0 \leq t \leq 1, \\
& \left.w\right|_{t=0}=\frac{1}{\pi} \arctan x+\frac{1}{2},\left.\quad \frac{\partial w}{\partial t}\right|_{t=0}=\frac{1}{\pi} \frac{\gamma}{1+x^{2}}, \\
& \left.w\right|_{t=1}=\frac{1}{\pi} \arctan (\gamma+x)+\frac{1}{2},\left.\quad \frac{\partial w}{\partial t}\right|_{t=1}=\frac{1}{\pi} \frac{\gamma}{1+(\gamma+x)^{2}},
\end{aligned}
$$

where $k>0, s>0$. Here we have

$$
\lambda(x)=\gamma+x, \quad R(x)=1, \quad S(x)=0 .
$$

Then the functional equation is $f(x)=f(x+\gamma)$ and it has many solutions because we obtain

$$
g(z)=\frac{\gamma}{\pi} \sin ^{2} \pi z\left(1-\frac{k}{\gamma} \sin 2 \pi z\right)-\left.f\left(\varphi_{0}^{-1}(z)\right) B \varphi_{0}(y)\right|_{y=\varphi_{0}^{-1}(z)} .
$$

If $f(x)=0$ then $g(z)=\frac{\gamma}{\pi} \sin ^{2} \pi z\left(1-\frac{k}{\gamma} \sin 2 \pi z\right)$. Namely, one of the solutions of the problem is

$$
\begin{aligned}
& w(x, t)=\frac{1}{\pi} \arctan (\gamma t+x)+\frac{1}{2}, \quad f(x)=0, \\
& g(z)=\frac{\gamma}{\pi} \sin ^{2} \pi z\left(1-\frac{k}{\gamma} \sin 2 \pi z\right) .
\end{aligned}
$$


Lemma 1 If the function $\lambda(x)$ is given in the special form

$$
\lambda(x)=\frac{x}{\left(1+\|x\|^{\alpha}\right)^{\frac{1}{\alpha}}},
$$

where $x \in E, \lambda(x) \in E, E$ is a Banach space, $\alpha>0$ is a fixed number, then we have

$$
\lambda^{[k]}(x)=\frac{x}{\left(1+k\|x\|^{\alpha}\right)^{\frac{1}{\alpha}}}, \quad k \geq 1 .
$$

Proof The lemma can be proved by a straightforward induction; that is, we assume

$$
\lambda^{[k]}(x)=\frac{x}{\left(1+k\|x\|^{\alpha}\right)^{\frac{1}{\alpha}}}
$$

and prove that

$$
\lambda^{[k+1]}(x)=\frac{x}{\left(1+(k+1)\|x\|^{\alpha}\right)^{\frac{1}{\alpha}}} .
$$

Thus, we can write

$$
\begin{aligned}
\lambda^{[k+1]}(x) & =\lambda\left(\lambda^{[k]}(x)\right) \\
& =\frac{\lambda^{[k]}(x)}{\left(1+\left\|\lambda^{[k]}(x)\right\|^{\alpha}\right)^{\frac{1}{\alpha}}} \\
& =\frac{\frac{x}{\left(1+k\|x\|^{\alpha}\right)^{\frac{1}{\alpha}}}}{\left(1+\left(\frac{\|x\|}{\left(1+k\|x\|^{\alpha}\right)^{\frac{1}{\alpha}}}\right)^{\alpha}\right)^{\frac{1}{\alpha}}} \\
& =\frac{\frac{x}{\left(1+k\|x\|^{\alpha}\right)^{\frac{1}{\alpha}}}}{\frac{\left(1+(k+1)\|x\|^{\alpha}\right)^{\frac{1}{\alpha}}}{\left(1+k\|x\|^{\alpha}\right)^{\frac{1}{\alpha}}}} \\
& =\frac{x}{\left(1+(k+1)\|x\|^{\alpha}\right)^{\frac{1}{\alpha}}} .
\end{aligned}
$$

\section{Corollary 1 If the relation}

$$
\varphi_{T}(x)=\varphi_{0}\left(\frac{x}{\left(1+|x|^{\alpha}\right)^{\frac{1}{\alpha}}}\right)
$$

holds, then we have

$$
\lambda(x)=\varphi_{0}^{-1}\left(\varphi_{T}(x)\right)=\frac{x}{\left(1+|x|^{\alpha}\right)^{\frac{1}{\alpha}}}
$$

and solution (17) can be written as

$$
f(x)=\sum_{k=0}^{\infty} \prod_{s=0}^{k-1} R\left(\frac{x}{\left(1+s|x|^{\alpha}\right)^{\frac{1}{\alpha}}}\right) S\left(\frac{x}{\left(1+k|x|^{\alpha}\right)^{\frac{1}{\alpha}}}\right) .
$$




\section{Inverse problem for a system of differential-difference equations}

As for the second problem of this paper, we will consider the system of differentialdifference equations

$$
w_{j}(x, t+h)=A_{j} w_{j}(x, t)+f_{j}(x) B_{j} w_{j}(x, t)+g_{j}\left(w_{1}(x, t), \ldots, w_{n}(x, t)\right)
$$

with the following data:

$$
\begin{array}{ll}
\left.w_{j}\right|_{t=a}=\varphi_{j a}(x), & w_{j}(x, a+h)=\psi_{j a}(x), \\
\left.w_{j}\right|_{t=b}=\varphi_{j b}(x), & w_{j}(x, b+h)=\psi_{j b}(x),
\end{array}
$$

where $j=1,2, \ldots, n$; $h$ is a constant, the operators $A_{j}, B_{j}$ act only on the variable $x,|x| \leq \tau$, $a \leq t \leq b$ and $w=\left(w_{1}, w_{2}, \ldots, w_{n}\right)$.

System (20) is important in modeling various social and economical problems. In particular, they arise in mathematical description of a fluctuating population of organisms, in control systems, and in economic studies of business cycles [7-9].

Problem 2 Determine the continuous functions $f_{j}(x)$ and $g_{j}(w)$ from system (20)-(21).

The result which we have obtained for Problem 2 is as follows.

Theorem 2 If there exist constants $q_{j} \in(0,1)$ and $\alpha_{j}>0$ such that

$$
\left|R_{j}(x)\right| \leq q_{j}, \quad\left|S_{j}(x)\right| \leq \alpha_{j}, \quad j=1,2, \ldots, n, x \in D,
$$

then the solution of the inverse problem (20)-(21) is given by the following formulas:

$$
\begin{aligned}
& f_{j}(x)=\sum_{k=0}^{\infty} \prod_{s=0}^{k-1} R_{j}\left(\lambda^{[s]}(x)\right) S_{j}\left(\lambda^{[k]}(x)\right), \\
& g_{j}(z)=\psi_{j a}\left(\varphi_{a}^{-1}(z)\right)-\left.A_{j} \varphi_{j a}(y)\right|_{y=\varphi_{a}^{-1}(z)}-\left.f_{j}\left(\varphi_{a}^{-1}(z)\right) B_{j} \varphi_{j a}(y)\right|_{y=\varphi_{a}^{-1}(z)} .
\end{aligned}
$$

Theorem 2 can be proved in the same way as in the proof of Theorem 1 .

We note that two important issues occur in studying the both inverse problems for equations (1) and (20). The first one is to investigate the related functional equation and the second one is to find the inverse mapping $x=\varphi_{a}^{-1}(y)$ on the domain $|x| \leq \tau,|y| \leq \tau$.

Example 3 Finally, let us consider the problem of determination of the functions $f(x)$ and $g(w)$ from the differential-difference equation

$$
w(x, t+h)=\frac{\partial^{2} w}{\partial x^{2}}+f(x)\left(\frac{\partial w}{\partial x}\right)^{2}+g(w), \quad 0 \leq x \leq \tau, 0 \leq t \leq T,
$$

and the data

$$
\begin{aligned}
& w(x, 0)=\varphi_{0}(x), \quad w(x, h)=\psi_{0}(x), \\
& w(x, T)=\varphi_{T}(x), \quad w(x, T+h)=\psi_{T}(x),
\end{aligned}
$$

where $0 \leq\left|\varphi_{0}(x)\right| \leq \tau, 0 \leq\left|\varphi_{T}(x)\right| \leq \tau$. 
Problem (22)-(23) can be reduced to a functional equation which is exactly the same as (16) in Example 1. Hence the solution can be obtained by (17)-(18).

\section{Conclusion}

In this paper, we have studied two inverse problems for systems of nonlinear differential and differential-difference evolution equations. First, we reduced the problems to some systems of functional equations by using the given data and then we obtained the solutions by the tools in [1]. We note that the proposed method can provide a new perspective for the theoretical and numerical study of inverse problems for such a kind of nonlinear systems.

\section{Competing interests}

The authors declare that they have no competing interests.

\section{Authors' contributions}

All authors contributed equally to the manuscript. All authors read and approved the final manuscript.

\section{Author details}

'Sobolev Institute of Mathematics, Siberian Branch of Russian Academy of Science, Acad. Koptyug prosp. 4, Novosibirsk, 630090 , Russia. ${ }^{2}$ Department of Mathematics, Faculty of Arts and Sciences, Bülent Ecevit University, Incivez, Zonguldak, 67100, Turkey.

\section{Acknowledgements}

The authors are grateful to anonymous referees for their constructive comments and suggestions, which have greatly improved this paper. This work is supported by Eren Enerji Elektrik Uretim A.S.

Received: 10 January 2016 Accepted: 26 May 2016 Published online: 10 June 2016

References

1. Kuczma, M: Functional Equations in a Single Variable. PWN, Warszawa (1968)

2. Debnath, L: Nonlinear Partial Differential Equations for Scientists and Engineers. Birkhäuser, Boston (2012)

3. Leung, AW: Nonlinear Systems of Partial Differential Equations: Applications to Life and Physical Sciences. World Scientific, Singapore (2009)

4. Zheng, S: Nonlinear Evolution Equations. Chapman \& Hall/CRC, Boca Raton (2004)

5. Anikonov, YE: Inverse Problems for Kinetic and Other Evolution Equations. VSP, Utrecht (2001)

6. Kuczma, M, Choczewski, B, Ger, R: Iterative Functional Equations. Cambridge University Press, Cambridge (1990)

7. Bellman, RE, Cooke, KL: Differential-Difference Equations. Academic Press, New York (1963)

8. Jones, GS: On the nonlinear differential-difference equation $f^{\prime}(x)=-\alpha f(x-1)\{1+f(x)\}$. J. Math. Anal. Appl. 4, 440-469 (1962)

9. Poluektov, RA, Pykh, YA, Shvytov, JA: Dynamical Models of Ecological Systems. Gidrometeoizdat, Leningrad (1980) (in Russian)

\section{Submit your manuscript to a SpringerOpen ${ }^{\circ}$ journal and benefit from:}

- Convenient online submission

Rigorous peer review

- Immediate publication on acceptance

Open access: articles freely available online

- High visibility within the field

- Retaining the copyright to your article 